\title{
LESÕES DESPORTIVAS E CUTÂNEAS EM ADEPTOS DE CORRIDA DE RUA
}

\author{
SPORTS INJURIES AND SKIN LESIONS IN ADEPTS OF STREET RACING \\ LESIONES DEPORTIVAS Y CUTÁNEAS EN ADEPTOS DE CARRERA DE CALLE
}

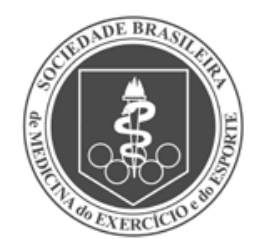

Artigo Original
Kátia Sheylla Malta Purim ${ }^{1,2}$ (Médica Dermatologista)

Ana Cláudia KappTitski²

(Educadora Física)

Paulo César Barauce Bento² (Educador Físico)

Neiva Leite² (Médica do Esporte)

1. Universidade Positivo,

Curitiba,PR, Brasil.

2. Universidade Federal do Paraná,

Curitiba,PR, Brasil.

\section{Correpondência:}

Comitê de Ética em Pesquisa da Universidade Positivo.

Rua Professor Pedro Viriato Parigot de Souza, 5300. Campo Comprido CEP 81280-330 Curitiba, PR, Brasil. kspurim@gmail.com

\begin{abstract}
RESUMO
Introdução: A corrida de rua é uma modalidade em expansão e tem sido utilizada como lazer, esporte ou prática competitiva. Objetivo: Verificar frequência de lesões desportivas e cutâneas em atletas de corrida de rua. Material e métodos: Estudo transversal de 220 atletas de corrida de rua com questionário e observação clínica. Análise pelos testes t, Mann-Whitney, Qui-quadrado e correlação de Spearman. Resultados: Predominaram atletas do sexo masculino, adultos, praticantes de corrida de longa distância, que treinam em média 45 a 60 minutos com frequência de 2 e 3 vezes por semana e têm participação variável em competições. As principais lesões relatadas foram dor no joelho (30,4\%), distensão/estiramento muscular $(16,7 \%)$, tendinites $(10,1 \%)$, dor nos quadris $(7,3 \%)$ e dor na coluna $(7 \%)$, sem diferenças estatísticas entre os gêneros. Houve associação significativa entre quilômetros percorridos em treino e lesões musculares nos homens ( $p=0,0477, x=9,60)$. O calçado esportivo teve relação com presença de unhas encravadas e perdas ungueais nas mulheres. Conclusão: Os homens apresentaram maior frequência de lesões musculares e as mulheres, de afecções cutâneas e ungueais.
\end{abstract}

Palavras-chave: traumatismos em atletas, corrida, dermatoses.

\section{ABSTRACT}

Introduction: Street racing is a sport in expansion and has been used as leisure, sport or competitive practice. Objective: To determine the frequency of sports injuries and skin lesions in athletes in street racing. Material and methods: Cross-sectional study of 220 athletes in street racing with questionnaire and clinical observation. Analysis by t test, Mann-Whitney, Chi-square and Spearman correlation. Results: There was a predominance of male athletes, adults, practitioners of distance running, training on average 45 to 60 minutes, 2 to 3 times a week and have variable participation in competitions. The main injuries reported were knee pain (30.4\%), ligament sprain/muscle strain (16.7\%), and tendinitis (10.1\%), pain in the hips (7.3\%) and back pain (7\%), with no significant differences between genders. There was a significant association between miles covered during training and muscle damage in men ( $p=0.0477, x=9.60)$. The athletic footwear was related to the presence of ingrown nails and nail loss in women. Conclusion: Men showed a higher frequency of muscle injuries and women had more skin and nail disorders.

Keywords: athletic injuries, running, skin diseases.

\section{RESUMEN}

Introducción: La carrera de calle es una modalidad en expansión y ha sido utilizada como recreación, deporte o práctica competitiva. Objetivo: Verificar frecuencia de lesiones deportivas y cutáneas en atletas de carrera de calle. Material y métodos: Estudio transversal de 220 atletas de carrera de calle con cuestionario y observación clínica. Análisis a través de los tests t, Mann-Whitney, Qui-cuadrado y correlación de Spearman. Resultados: Predominaron atletas del sexo masculino, adultos, practicantes de carrera de larga distancia, que entrenan como promedio 45 a 60 minutos con frecuencia de 2 y 3 veces por semana y tienen participación variable en competiciones. Las principales lesiones relatadas fueron dolor en la rodilla (30,4\%), distensión/estiramiento muscular $(16,7 \%)$, tendinitis $(10,1 \%)$, dolor en los muslos $(7,3 \%)$ y dolor en la columna (7\%), sin diferencias estadísticas entre los géneros. Hubo asociación significativa entre kilómetros recorridos en entrenamiento y lesiones musculares en los hombres ( $p=0,0477, x=9,60)$. El calzado deportivo tuvo relación con presencia de uñas encarnadas y pérdidas ungueales en las mujeres. Conclusión: Los hombres presentaron mayor frecuencia de lesiones musculares y las mujeres, de afecciones cutáneas y ungueales.

Palabras clave: traumatismos en atletas, carrera, dermatosis. 


\section{INTRODUÇÃO}

O exercício físico regular é associado à aptidão física, bem estar e qualidade de vida'. A corrida de rua tem sido utilizada como lazer, esporte, reabilitação ou prática competitiva, por ser opção de fácil acesso, de baixo custo e capaz de produzir múltiplos benefícios para os diversos sistemas corporais ${ }^{2-4}$. Como toda prática esportiva, a corrida expõe o praticante a vários fatores que propiciam o aparecimento de lesões. O conceito de lesão esportiva apresenta grande variabilidade na literatura e, em geral, pressupõe afecção musculoesquelética, dor ou incapacidade para treinos e/ou competições ${ }^{4-6}$.

A pele compõe o sistema imunológico de defesa periférica, sendo o maior órgão do corpo humano diretamente exposto a agressões associadas às atividades esportivas ${ }^{7}$. A propriedade de barreira da pele varia com a espessura, a permeabilidade, a região anatômica e a capacidade de reagir imunologicamente. A resposta cutânea em geral está condicionada a condições predisponentes como idade, sexo, etnia, clima, antecedentes mórbidos, características das atividades físicas, treinamento e competição. Agentes físicos, químicos, biológicos e ergonômicos também podem causar distúrbios tegumentares entre os praticantes de corrida ${ }^{8,9}$. Porém há poucos dados na literatura sobre as lesões cutâneas associadas a esta atividade física. As lesões dermatológicas merecem atenção, pois a barreira cutânea trata-se do primeiro mecanismo de separação entre corpo e meio ambiente, motivo pelo qual está constantemente sob o efeito de injúrias $7,10,11$.

As alterações musculoesqueléticas são as mais descritas entre os praticantes de corrida e incluem fraturas, luxações, distensões musculares e entorses ${ }^{3,5,6}$. A maioria destas condições está intimamente ligada a erros de técnica, alongamento e aquecimento insuficientes, bem como a equipamentos inadequados e falta de acompanhamento médico ${ }^{5}$. Entretanto, as lesões cutâneas no atleta podem ocorrer em conjunto com os danos ortopédicos, geralmente resultantes de traumas, infecções, exacerbações de condições pré-existentes e fatores ambientais, como a exposição à luz solar, a constante fricção da pele contra os materiais de treino (calçados e vestuário) e o impacto no solo ${ }^{12}$.

Nos praticantes de esporte ao ar livre tem sido relatados hematomas, escoriações, eritema, edema, bolhas e queimaduras na pele decorrente da exposição ambiental desprotegida e aos microtraumas causados pelo atrito dos calçados, roupas e demais equipamentos ${ }^{7,13-15}$. O ar frio, névoas, garoa, chuva e a poluição ambiental, além da radiação ultravioleta, podem isolados ou simultaneamente provocar reações cutâneas irritativas, inflamatórias e neoplásicas ${ }^{9,16}$. Durante a corrida, o suor gera aumento da perda transepidérmica de água tornando a pele mais ressecada e vulnerável a riscos e danos, podendo apresentar amplo espectro clínico de lesões iniciadas, mantidas ou agravadas pelo esporte ${ }^{9,11}$.

Portanto, estudos sobre a prevalência das lesões dermatológicas em corredores ainda são escassos, limitando-se, na maioria dos casos, a uma modalidade esportiva específica, o que não permite o aproveitamento dos dados para os corredores de forma absoluta. Tais estudos, associados à crescente adesão a pratica da corrida, justificam e reforçam a necessidade de dados específicos, direcionados para esta modalidade esportiva e seus atletas, tanto profissionais quanto amadores. O trabaIho diagnóstico e preventivo de lesões dermatológicas na preparação e acompanhamento dos atletas poderá minimizar perda de desempenho esportivo ${ }^{17}$. O objetivo desta pesquisa foi avaliar a prevalência de lesões desportivas e cutâneas em praticantes de corrida de rua da cidade de Curitiba - PR, Brasil.

\section{MATERIAL E MÉTODO}

Estudo epidemiológico, descritivo e transversal, tendo como população alvo os atletas inscritos em evento competitivo de meia ma- ratona realizado na cidade de Curitiba (PR) na região sul do Brasil em setembro de 2012. Participaram deste estudo indivíduos de ambos os gêneros, maiores de 18 anos, independente da etnia, integrante de grupo orientado de corrida há pelo menos seis meses. O projeto foi aprovado por Comitê de Ética (CEP-UP 055/2011) e a inclusão dos atletas ocorreu mediante adesão voluntária e consentimento escrito, conforme resolução 196/96.

Os dados foram coletados por meio de questionário com validação de conteúdo, composto de questões fechadas que abordavam as características sociodemográficas dos atletas como: sexo, idade, cor da pele, profissão, escolaridade, dados de estilo de vida e da prática esportiva. O treinamento foi avaliado quanto ao tempo de prática da corrida de rua (em anos), frequência e duração dos percursos semanais, velocidade média dos treinos (quilômetro/hora) e participação em competições (municipal, estadual, nacional, internacional). Em relação aos tênis esportivos investigou-se o tempo de permanência do calçado, quantidade de tênis específicos para corrida e sua substituição diária e em meses, o número do calçado utilizado (menor, maior ou igual ao número do calçado não esportivo) e uso ou não de protetores nos pés (palmilhas ou proteção para calos).

Para verificar as lesões musculoesqueléticas, afecções cutâneas nos membros inferiores e tratamentos cirúrgicos relacionados ao esporte utilizou-se recordatório de dados clínicos esportivos. O questionário foi aplicado no momento da entrega do número de inscrição (kit participação) relacionado à corrida por equipe previamente treinada.

Para a análise da prevalência de lesões desportivas e cutâneas, a amostra foi dividida em grupo masculino e grupo feminino. Para fins deste estudo, consideramos lesão desportiva qualquer dor, afecção ou agravo que tenha limitado ou afastado por um ou mais dias a participação do atleta em treinos e/ou competições nos últimos seis meses $^{18}$. Estas lesões foram subdivididas em três grupos: musculares, osteoarticulares (dor no joelho, no quadril, nas costas, nos pés) e tendinopatias, sendo verificadas suas características nos diferentes volumes de treino e gênero.

Definiu-se como treino de baixo volume semanal os participantes que corriam até $24 \mathrm{~km}$ semanais, médio volume semanal de 24 a 49 $\mathrm{km}$ semanais, e alto volume semanal acima de $50 \mathrm{~km}$ semanais.

Os resultados foram analisados com base em estatística descritiva, sendo agrupados em média e desvio-padrão. Para análise da normalidade dos dados, foi o utilizado o teste Kolmogorov-Smirnov. O teste $t$, para as variáveis normais, e o teste de Mann-Whitney, para as não normais, foram utilizados para comparação das variáveis entre os gêneros. Para avaliar o perfil de sintomas e lesões dos atletas em função do percurso semanal treinado, realizou-se o teste de Qui-quadrado e correlação de Spermann. Foram realizadas estatísticas inferências de associação para a determinação dos fatores de risco para ocorrência de lesões de pele. Para tal, foi aplicado o teste de Qui-quadrado. Em todos os testes foi estabelecido nível de significância com $\mathrm{p}<0,05$.

\section{RESULTADOS}

\section{Perfil dos praticantes de corrida de rua}

A amostra foi composta por 220 praticantes regulares de corrida de rua que participaram em evento competitivo em setembro de 2012 na cidade de Curitiba, PR, Brasil. Destes indivíduos, 75,4\% eram do gênero masculino e $24,6 \%$ do gênero feminino. Predominavam desportistas da raça branca (74\%), procedentes do sul do Brasil (85,9\%), exercendo diversas profissões ligadas à indústria e o comércio, sendo que 81,8\% apresentavam instrução em ensino superior ou mais. 


\section{Caracterização dos dados esportivos}

Os participantes praticavam a modalidade de corrida de longa distância, sendo que 29,5\% dos atletas praticam a atividade a um período de 1 a 3 anos, 40\% a um período de 4 a 9 anos e 19,6\% praticam a mais de 10 anos, sem diferenças significativas entre os gêneros (tabela 1).

Aproximadamente $73,6 \%$ correm por semana percursos que variam de 12 a 49 km, sendo que 26,4\% correm mais de $50 \mathrm{~km}$ por semana. A maioria destes atletas (71,3\%) investe em média 45 a 60 minutos por treino, com frequências semanais variáveis de 2 e 3 vezes por semana $(66,3 \%)$ ou 4 a 7 vezes por semana $(33,7 \%)$ (tabela 2$)$.

\section{Participação em competições}

Aproximadamente $64,8 \%$ dos analisados correm apenas em competições municipais ou estaduais e apenas 7,3\% correm em todas as competições. Contudo, vale ressaltar que do total da amostra 41,3\% correm nas competições municipais, 23,5\% em competições estaduais, 25,2\% em competições nacionais e 10\% em competições internacionais.

A frequência com que participam de competições é bastante variável, sendo que aproximadamente 17,8\% dos entrevistados participam de uma corrida ao mês. Grande parte dos sujeitos analisados pratica seus treinamentos na rua $(54,1 \%)$, mas combinam seus treinos em pistas, esteiras, estradas de chão e grama.

\section{Caracterização das lesões desportivas}

Sobre a caracterização das lesões desportivas, 65,9\% dos praticantes de corrida de rua já tiveram lesões músculo-ortopédicas limitantes e que impediram à atividade esportiva. Dentre os atletas que já apresentaram tais limitações, os principais tipos de lesões descritos foram: distensão/estiramento muscular (16,7\%), tendinites (10,1\%), dor nos quadris (7,3\%) e dor na coluna (7\%). A dor no joelho acometeu 67

Tabela 1. Características descritivas da amostra.

\begin{tabular}{|c|c|c|c|c|c|c|c|}
\hline Variáveis & \multicolumn{2}{|c|}{$\begin{array}{c}\text { Total } \\
(n=220)\end{array}$} & \multicolumn{2}{|c|}{$\begin{array}{l}\text { Homens } \\
(n=166)\end{array}$} & \multicolumn{2}{|c|}{$\begin{array}{c}\text { Mulheres } \\
(n=54)\end{array}$} & Valor $p$ \\
\hline Idade & \multicolumn{2}{|c|}{$38,4 \pm 11,3$} & \multicolumn{2}{|c|}{$38,9 \pm 11,2$} & \multicolumn{2}{|c|}{$36,7 \pm 11,5$} & 0,212 \\
\hline Peso & \multicolumn{2}{|c|}{$71,09 \pm 12,46$} & \multicolumn{2}{|c|}{$74,55 \pm 10,74$} & \multicolumn{2}{|c|}{$60,51 \pm 11,42$} & $0,000^{*}$ \\
\hline Estatura & \multicolumn{2}{|c|}{$1,72 \pm 0,08$} & \multicolumn{2}{|c|}{$1,74 \pm 0,07$} & \multicolumn{2}{|c|}{$1,63 \pm 0,06$} & $0,000^{*}$ \\
\hline $\mathrm{IMC}$ & \multicolumn{2}{|c|}{$23,95 \pm 3,08$} & \multicolumn{2}{|c|}{$24,41 \pm 2,96$} & \multicolumn{2}{|c|}{$22,5 \pm 3,03$} & $0,000^{*}$ \\
\hline \multicolumn{8}{|c|}{ Cor da pele } \\
\hline Branca & 163 & $74 \%$ & 119 & $71,7 \%$ & 44 & $81,4 \%$ & \\
\hline Parda & 30 & $13,6 \%$ & 24 & $13,8 \%$ & 6 & $11,1 \%$ & \\
\hline Amarela & 13 & $5,9 \%$ & 12 & $7,2 \%$ & 1 & $1,8 \%$ & \\
\hline Preta & 11 & $5 \%$ & 8 & $4,8 \%$ & 3 & $5,5 \%$ & 0,4137 \\
\hline \multicolumn{8}{|c|}{ Escolaridade } \\
\hline Fundamental & 3 & $1,3 \%$ & 3 & $1,8 \%$ & 0 & $0 \%$ & \\
\hline $\begin{array}{c}\text { Médio } \\
\text { Incompleto }\end{array}$ & 4 & $1,9 \%$ & 4 & $2,4 \%$ & 0 & $0 \%$ & \\
\hline Médio & 33 & $15,1 \%$ & 29 & $17,4 \%$ & 4 & $7,4 \%$ & \\
\hline $\begin{array}{c}\text { Superior } \\
\text { incompleto }\end{array}$ & 30 & $13,6 \%$ & 21 & $12,6 \%$ & 9 & $16,6 \%$ & \\
\hline $\begin{array}{l}\text { Superior } \\
\text { completo }\end{array}$ & 67 & $30,4 \%$ & 50 & $30,1 \%$ & 17 & $31,4 \%$ & \\
\hline Pós-graduação & 83 & $37,7 \%$ & 59 & $35,5 \%$ & 24 & $44,4 \%$ & 0,2701 \\
\hline \multicolumn{8}{|c|}{ Tempo de treinamento } \\
\hline Menos de 1 ano & 25 & $10,9 \%$ & 17 & $10,2 \%$ & 8 & $14,8 \%$ & \\
\hline 1 a 3 anos & 65 & $29,5 \%$ & 47 & $28,3 \%$ & 18 & $33,3 \%$ & \\
\hline 4 a 6 anos & 62 & $28,2 \%$ & 46 & $27,7 \%$ & 16 & $29,6 \%$ & \\
\hline 7 a 9 anos & 26 & $11,8 \%$ & 21 & $12,6 \%$ & 5 & $0,9 \%$ & \\
\hline 10 a 15 anos & 21 & $9,5 \%$ & 19 & $11,4 \%$ & 2 & $3,7 \%$ & \\
\hline 16 a 20 anos & 7 & $3,3 \%$ & 6 & $3,6 \%$ & 1 & $1,8 \%$ & \\
\hline $\begin{array}{c}\text { Acima de } \\
20 \text { anos }\end{array}$ & 15 & $6,8 \%$ & 11 & $6,6 \%$ & 4 & $7,4 \%$ & 0,6023 \\
\hline
\end{tabular}

Tabela 2. Características de treinamento semanal e participação em competições.

\begin{tabular}{|c|c|c|c|c|c|c|c|}
\hline Variáveis & \multicolumn{2}{|c|}{$\begin{array}{c}\text { Total } \\
(n=220)\end{array}$} & \multicolumn{2}{|c|}{$\begin{array}{c}\text { Masculino } \\
(n=166)\end{array}$} & \multicolumn{2}{|c|}{$\begin{array}{c}\text { Feminino } \\
(n=54)\end{array}$} & Valor $p$ \\
\hline \multicolumn{8}{|c|}{ Frequência semanal de treinos } \\
\hline & $\mathrm{n}$ & $\%$ & $\mathrm{n}$ & $\%$ & $\mathrm{n}$ & $\%$ & \\
\hline 3 & 79 & $35,9 \%$ & 57 & $34,3 \%$ & 22 & $40,7 \%$ & \\
\hline 4 & 67 & $30,4 \%$ & 52 & $31,3 \%$ & 15 & $27,7 \%$ & \\
\hline 5 & 35 & $15,9 \%$ & 29 & $17,4 \%$ & 6 & $11,1 \%$ & \\
\hline 6 & 38 & $17,3 \%$ & 27 & $16,3 \%$ & 11 & $20,4 \%$ & 0,5603 \\
\hline \multicolumn{8}{|c|}{ Distância média semanal } \\
\hline$<12,12$ a $24 \mathrm{~km}$ & 77 & $35 \%$ & 53 & $31,9 \%$ & 24 & $44,4 \%$ & \\
\hline 25 a $49 \mathrm{~km}$ & 85 & $38,6 \%$ & 68 & $40,9 \%$ & 17 & $31,5 \%$ & \\
\hline 50 a 64 km & 30 & $13,6 \%$ & 22 & $13,2 \%$ & 8 & $14,8 \%$ & \\
\hline 65 a 94 & 15 & $6,8 \%$ & 13 & $7,8 \%$ & 2 & $3,7 \%$ & \\
\hline$>95 \mathrm{~km}$ & 13 & $5,9 \%$ & 10 & $6 \%$ & 3 & $5,5 \%$ & 0,4227 \\
\hline \multicolumn{8}{|c|}{ Duração treino } \\
\hline $45 \mathrm{~min}$ & 44 & $20 \%$ & 29 & $17,4 \%$ & 15 & $27,7 \%$ & \\
\hline $60 \mathrm{~min}$ & 115 & $52,3 \%$ & 91 & $54,8 \%$ & 24 & $44,4 \%$ & \\
\hline 1 hora 30 minutos & 40 & $18,2 \%$ & 30 & $18 \%$ & 10 & $18,5 \%$ & \\
\hline 2 horas & 14 & $6,4 \%$ & 10 & $6 \%$ & 4 & $7,4 \%$ & 0,3781 \\
\hline \multicolumn{8}{|c|}{ Participação em competições } \\
\hline Municipal & 128 & $58,2 \%$ & 90 & $54,1 \%$ & 38 & $70,4 \%$ & \\
\hline Estadual & 73 & $33,2 \%$ & 58 & $34,9 \%$ & 15 & $27,7 \%$ & \\
\hline Nacional & 78 & $35,4 \%$ & 57 & $34,3 \%$ & 21 & $38,9 \%$ & \\
\hline Internacional & 31 & $14 \%$ & 22 & $13,2 \%$ & 9 & $16,6 \%$ & 0,5562 \\
\hline
\end{tabular}

atletas (30,4\%), sendo 54 (32,5\%) do gênero masculino e 13 (44\%) do gênero feminino, sem diferença significativa ( $p=0,242)$ (tabela 3).

Em relação a características das lesões desportivas não foram observadas associações significativas com os quilômetros de treino, porém quando analisados os gêneros, houve associação significativa somente nas lesões musculares nos homens ( $p=0,0477, x=9,603$ ).

Nas afecções dermatológicas, os atletas apresentavam nos pés: calos $(35,6 \%)$, bolhas $(32,1 \%)$, perdas de unhas $(31,5 \%)$, unha encravada (17,3\%) e micoses (10,9\%). Nas outras regiões cutâneas, destacavam-se assaduras $(30,9 \%)$, queimaduras solares $(16,4 \%)$, rachadura nos lábios $(6,5 \%)$ e lesões nos mamilos (4,6\%). Não houve diferença significativa entre os calos, bolhas e micoses nos pés entre os gêneros (tabela 4).

\section{Características dos calçados esportivos utilizados}

Em relação às características dos calçados utilizados por estes atletas, a maioria usa de 1 a 3 tênis (86,9\%) para as corridas, sendo que predominou a substituição de calçados em um período variável de 3 a

Tabela 3. Características das lesões desportivas.

\begin{tabular}{|c|c|c|c|c|c|c|c|c|c|c|c|}
\hline \multirow[b]{2}{*}{ Musculares } & \multicolumn{2}{|c|}{$\begin{array}{c}12 \text { a } 24 \\
\text { km }\end{array}$} & \multicolumn{2}{|c|}{$\begin{array}{c}25 \text { a } 49 \\
\mathrm{~km}\end{array}$} & \multicolumn{2}{|c|}{$\begin{array}{c}50 \text { a } 64 \\
\mathrm{~km}\end{array}$} & \multicolumn{2}{|c|}{$\begin{array}{c}65 \text { a } 94 \\
\mathrm{~km}\end{array}$} & \multicolumn{2}{|c|}{$>95 \mathrm{~km}$} & \multirow{2}{*}{$\begin{array}{c}\mathbf{P} \\
0,2371 \\
\end{array}$} \\
\hline & 11 & $14,3 \%$ & 21 & $24,7 \%$ & 8 & $26,6 \%$ & 3 & $20 \%$ & 5 & $38,4 \%$ & \\
\hline Osteoarticulares & 57 & $74 \%$ & 62 & $72,9 \%$ & 26 & $86,6 \%$ & 14 & $93,3 \%$ & 9 & $69,3 \%$ & 0,2571 \\
\hline Tendinopatias & 6 & $7,8 \%$ & 11 & $12,9 \%$ & 5 & $16,6 \%$ & 3 & $20 \%$ & 4 & $30,7 \%$ & 0,2168 \\
\hline
\end{tabular}

Tabela 4. Características das lesões cutâneas mais freqüentes nos pés.

\begin{tabular}{c|c|c|c|c|c|c|c}
\hline Variáveis & \multicolumn{2}{|c|}{$\begin{array}{c}\text { Total } \\
(\mathbf{n = 2 2 0 )}\end{array}$} & \multicolumn{2}{c|}{$\begin{array}{c}\text { Masculino } \\
(\mathbf{n = 1 6 6 )}\end{array}$} & \multicolumn{2}{c|}{$\begin{array}{c}\text { Feminino } \\
(\mathbf{n = 5 4 )}\end{array}$} & Valor $\mathbf{p}$ \\
\hline & $\mathbf{n}$ & $\%$ & $\mathbf{n}$ & $\%$ & $\mathbf{n}$ & $\%$ & \\
\hline Calos & 78 & $35,4 \%$ & 54 & $32,5 \%$ & 24 & $44,4 \%$ & 0,1119 \\
\hline Bolhas & 98 & $44,5 \%$ & 71 & $42,7 \%$ & 27 & $50 \%$ & 0,353 \\
\hline Perdas de unhas & 69 & $31,3 \%$ & 46 & $27,7 \%$ & 23 & $42,6 \%$ & 0,040 \\
\hline Unha encravada & 38 & $17,3 \%$ & 24 & $14,4 \%$ & 14 & $25,9 \%$ & 0,052 \\
\hline Micoses & 33 & $15 \%$ & 27 & $16,2 \%$ & 6 & $11,1 \%$ & 0,3569 \\
\hline Teste Qui-quadrado. & & & & &
\end{tabular}


10 meses (64\%). O tempo de permanência com o tênis de corrida dura 1 a 3 horas diárias (81\%), e normalmente os atletas utilizam o mesmo número $(50,2 \%)$ ou um número maior do que o número do calçado habitual (46,5\%). Parte da amostra assumiu situações de desconforto com o uso do tênis (22,9\%), como dores e lesões $(77,1 \%)$, contudo apenas 44,9\% utilizavam recursos específicos para proteção dos pés (palmilha, protetor de calo) durante os treinamentos.

O tamanho do tênis utilizado demonstrou significância com presença de unha encravada e perda de unha em relação aos gêneros. Nas mulheres, a unha encravada $(r=0,359, p=0,008)$ e a perda de unha $(r=-0,318, p=0,019)$ apresentaram correlação inversa e significativa com o tamanho do tênis igual ao calçado habitual. Enquanto que, a relação destas lesões com o calçado maior que o habitual apresentou correlação direta e significativa com a unha encravada $(r=0,338, p=0,012)$ e perda de unha ( $r=0,337, p=0,013$ ). Estas diferenças não foram estatisticamente significativas nos homens.

\section{Distância percorrida e presença de lesões}

Ao avaliar o padrão de dores dos participantes, foi possível verificar que todos os grupos apresentaram as mesmas proporções observadas no levantamento geral da amostra ( $p>0,05)$. Não foram observadas diferenças entre os percursos semanais e queixas de dores nos pés, tornozelos, joelhos, calcanhares e coluna. A amostra de atletas que percorre mais de $95 \mathrm{~km}$ também manifestou dores nos joelhos $(61,5 \%)(p=0,000)$.

Em relação aos problemas tendíneo-musculares e cirúrgicos, observou-se que todos os grupos apresentam homogeneidade na distribuição das freqüências, sem diferenças em relação à quilometragem semanal percorrida.

As unhas encravadas foram mais frequentes entre os participantes que percorriam acima de $95 \mathrm{~km}(\mathrm{p}=0,016)$. As infecções micóticas nos pés foram mais frequentes entre os desportistas que corriam mais de 50 km por semana ( $p=0,014)$. Sobre as demais queixas cutâneas não houve diferenças estatísticas entre os grupos e o percurso.

\section{DISCUSSÃO}

O interesse e a participação de homens e mulheres em corridas de rua têm aumentado, incluindo jovens e idosos, que buscam melhorar condicionamento físico e qualidade de vida ${ }^{1,4}$. Entretanto, as lesões desportivas cutâneas podem interferir no treinamento e no desempenho esportivo, prejudicando a participação em atividades recreativas ou competitivas, devido dor, desconforto, limitação funcional e infecções ${ }^{11,19}$.

Este estudo visou estabelecer qual a prevalência destas lesões na modalidade de corrida de rua praticada por 220 atletas no município de Curitiba. Entre os participantes, houve predominância de homens brancos, adultos entre a terceira e quarta década de vida, com boa escolaridade, que praticavam corrida de rua de longa distância. Na composição da amostra, observaram-se diferenças significativas entre homens e mulheres para peso, estatura e IMC, características antropométricas relacionadas ao gênero. Houve discreta tendência de maior escolaridade entre as mulheres. Quanto à caracterização dos dados desportivos, a amostra mostrou-se homogênea quando comparados os gêneros.

A corrida de rua tem se popularizado pela facilidade, baixo custo, acoIhimento aos diferentes tipos de desportistas, crescente número de provas e eventos competitivos ${ }^{4,10,20}$. Nesta pesquisa, houve maior participação das mulheres nas competições municipais $(70,4 \%)$. As possíveis justificativas para a predominância feminina em eventos locais são o tempo limitado para deslocamentos para competições, devido às múltiplas jornadas de trabalho das mulheres, bem como a responsabilidade nas tarefas domésti$\operatorname{cas}^{21}$. Nesta amostra, o grupo masculino apresentava maior disponibilidade de deslocamento para competições estaduais, nacionais e internacionais.

Neste estudo, não foram observadas diferenças significativas nas frequências das lesões musculares, osteoarticulares etendinopatias em relação à distância percorrida, resultados concordantes com outras pesquisas desenvolvidas em praticantes de corrida de rua $a^{3,4}$ e em adeptos de atletismo ${ }^{5}$. Na comparação entre os gêneros, houve predomínio de dor muscular nos homens, possivelmente associado a maior participação em competições. Pesquisas apontam que os homens se engajam mais em atividades físicas de caráter competitivo ${ }^{21}$.

Quanto à localização das lesões dermatológicas, houve maior prevalência de acometimento de membros inferiores em concordância com a literatura ${ }^{9,16}$. No presente estudo não foram encontradas diferenças significativas entre os gêneros para calos e bolhas. Houve predomínio de unhas encravadas e perdas de unhas nas mulheres, com diferenças significativas entre os gêneros. Provavelmente, aspectos anatômicos do pé feminino e hábitos cosméticos de embelezamento das unhas podem ser fatores associados ao calçado mal adaptado que se constitui em importante agente causal dos distúrbios ungueais ${ }^{22,23}$.

O uso de calçados inadequados, os movimentos rápidos, os diferentes tipos de superfície e solos, bem como a falta de sinergismo muscular são fatores que favorecem o surgimento de lesões, especialmente, em membros inferiores ${ }^{12,15,19,24}$. A resposta cutânea estará condicionada a fatores raciais, genéticos, dermatoses pré-existentes, como ainda ao tempo e intensidade da ação mecânica do calçado, podendo provocar danos agudos ou crônicos ${ }^{17}$.

A importância destas dermatoses é o risco de desajustes no posicionamento corporal, o que afeta o desempenho esportivo negativamente, aumentando também a probabilidade de acarretar lesões no futuro em especial pelas sobrecargas excessivas sobre os pés $s^{9,12,17}$ e influência de fatores ergonômicos como força, repetitividade, posturas inadequadas e compressão mecânica ${ }^{23,24}$.

Pesquisas mostram aumento de lesões musculoesqueléticas entre corredores, conforme o incremento do volume semanal de treinos e quilometragem percorrida ${ }^{4,8,25}$. Nesta investigação, $65,9 \%$ dos praticantes de corrida de rua relataram lesões músculo-ortopédicas limitantes e que impediram à atividade esportiva, com principal destaque para a articulação do joelho, que apresentou manifestação dolorosa proporcional ao aumento da quilometragem semanal percorrida. De acordo com Yeung e Yeung ${ }^{26}$, há evidências científicas de que a redução na distância percorrida pode reduzir o surgimento de lesões. Em contrapartida, o estudo de Pazin et al. ${ }^{3}$ demonstrou que o maior tempo de prática em anos não está significativamente associado à prevalência de lesão.

A corrida de rua requer condições específicas de treinamento direcionado para atividades físicas de longa duração, moderada a intensa com utilização de grandes grupos musculares, que envolve elementos básicos da corrida, adaptados ao atleta, clima, piso da prática esportiva e evento disputado ${ }^{5,6}$. Neste estudo, os atletas sofreram distintas lesões seja na iniciação da modalidade ou em altos níveis de desempenho. Entretanto, abordando mais especificamente os resultados obtidos na comparação entre os gêneros, observou-se que as taxas de lesões ungueais foram mais elevadas entre as mulheres.

Também foi significativo o registro de lesões desportivas nas muIheres que corriam percursos menores de $25 \mathrm{~km}$ ou maiores de $95 \mathrm{~km}$. Entre as justificativas, estão as peculiaridades biomecânicas, hormonais e neuromusculares femininas que provavelmente influenciaram a ocorrência destas lesões, bem como, aspectos diretos ou indiretos relacionados à preparação e prática da corrida $4,27,28$. Outro fato relacionado é a experiência masculina nas competições e adaptações a maiores distâncias ${ }^{3}$, que pode minimizar a frequência das lesões desportivas.

Muitas das lesões em membros inferiores podem ser prevenidas se atenção especial for direcionada para o uso de equipamentos 
esportivos adequados, reconhecimento precoce do problema, realização de inspeção periódica da pele e acompanhamento médico.

As limitações deste estudo são o delineamento que não permite inferir causalidade e o reduzido tamanho amostral. Entretanto, a partir das observações e comparações realizadas, esta pesquisa identificou importantes diferenças entre os gêneros na participação em competições e presença de lesões desportivas e cutâneas, com elevada taxa de agravos no público feminino. Sendo assim as características do treinamento feminino precisam ser analisadas para que esta prática esportiva possa ser mais segura e satisfatória, visando obter melhor qualidade de vida através deste esporte.

\section{CONCLUSÕES}

Agravos musculoesqueléticos nos joelhos, tornozelos e pés foram frequentes nos praticantes de corrida. As dermatoses mais comuns foram calos e distúrbios ungueais nos pés. Homens e mulheres apresentaram lesões desportivas e cutâneas distintas associadas a este esporte, sendo que os homens apresentaram maior frequência de lesões musculares e as mulheres as afecções cutâneas e ungueais.

Todos os autores declararam não haver qualquer potencial conflito de interesses referente a este artigo.

\section{REFERÊNCIAS}

1. Garber CE, Blissmer B, Deschenes MR, Franklin BA, Lamonte MJ, Lee IM, et al. Quantity and quality of exercise for developing and maintaining cardiorespiratory, musculoskeletal, and neuromotor fitness in apparently healthy adults: guidance for prescribing exercise. Med Sci Sports Exerc. 2011;43(7):1334-59

2. IAAF. Associação Internacional das Federações de Atletismo. Disponível em: http://www.iaaf.org (Acesso em 10 abril 2013).

3. Pazin J, Duarte MFS, Poeta LS, Gomes MA. Corredores de rua: características demográficas, treinamento e prevalência de lesões. Rev Bras Cineantropom Desempenho Hum. 2008; 10(3):277-82.

4. Ferreira AC, Dias JMC, Fernandes RM, Sabino GS, Anjos MTS, Felício DC. Prevalência e fatores associados a lesões em corredores de rua do município de Belo Horizonte, MG. Rev Bras Med Esporte. 2012;18(4): 252-5

5. Pastre $C M$, Carvalho Filho G, Monteiro HL, Netto Junior J, Padovani $C R$, Garcia $A B$. Exploração de fatores de risco para lesões no atletismo de alta performance. Rev Bras Med Esporte. 2007;13(3):200-4.

6. Pastre $\mathrm{CM}$, Carvalho Filho $\mathrm{G}$, Monteiro $\mathrm{HL}$, Netto Junior J, Pandovani $\mathrm{CR}$. Lesões desportivas na elite do atletismo brasileiro: estudo a partir de morbidade referida. Rev Bras Med Esporte. 2005;11(1):43-7.

7. Heymann WR. Dermatologic problems of the endurance athlete. J Am Acad Dermatol. 2005; 52: 345-6.

8. Fredericson M, Misra AK. Epidemiology and an etiology of marathon running injuries. Sports Med. 2007;37(4-5):437-9.

9. Mailler EA, Adams BB. The wear and tear of 26.2 dermatological injuries reported on marathon day. Br J Sports Med. 2004;38(4):498-501.

10. Brunet ME, Cook SD, Brinker MR, Dickinson JA. A survey of running injuries in 1505 competitive and recreational runners. J Sports Med Phys Fitness. 1990;30(3): 307-15.

11. Bender TW. Cutaneous manifestations of disease in athlete. Skinmed. 2003:2(1):34-41.

12. Purim KSM, Niehues LP, Queiroz-Telles FF, Leite N. Aspectos epidemiológicos das micoses dos pés em um time chinês de futebol. Rev Bras Med Esporte. 2006;12(1):16-9.

13. Pecci M, Comeau D, Chawla V. Skin conditions in the athlete. Am J Sports Med. 2009;37(2):406-18.

14. Metelitsa A, Barankin B, Lin AN. Diagnosis of sports related dermatoses. Int J Dermatol. 2004;43(2):113-93.
15. Luca JF, Adams BB, Yosipovitch $G$. Skin manifestations of athletes competing in the summer olympics: what a sports medicine physician should know. Sports Med. 2012;42(5):399-413.

16. Mailler-Savage EA, Adams BB. Skin manifestations of running. J Am Acad Dermatol. 2006;55(2):290-301.

17. Basler RSW, Hunzeker CM, Garcia MA. Athletic skin injuries: combating pressure and friction. Phys Sports Med. 2004;32(5):1-9.

18. Lun V, Meeuwisse WH, Stergiou P. Stefanyshyn D. Relation between running injury and static lower limb alignment in recreational runners. Br J Sports Med. 2004;38(5):576-80.

19. Helm MF, Helm TN, Bergfeld WF. Skin problems in the long-distance runner 2500 years after the Battle of Marathon. Int J Dermatol. 2012;51(3):263-70.

20. Salgado JVV, Chacon-Mikahil MPT. Corrida de rua: análise do crescimento do número de provas e de praticantes. Conexões. 2006;4:90-9.

21. Salles-Costa R, Heilborn ML, Werneck GL, Faerstein E, Lopes CS. Gênero e prática de atividade física de lazer. Cad Saúde Pública. 2003;19(2):325-33.

22. Oumeish YO. Common acrally distributed dermatoses. Clin Dermatol. 2011;29(2):130-9.

23. Richards CE, Magin PJ, Callister R. Is your prescription of distance running shoes evidence-based? $\mathrm{Br}$ J Sports Med. 2009;43(3):159-62

24. Cowan DN, Jones BH, Robinson JR. Foot morphologic characteristics and risk of exercise-related injury. Arch Fam Med. 1993:2(7):773-7.

25. Hootman JM, Macera CA, Ainsworth BE, Addy CL, Martin M, Blair SN. Epidemiology of musculoskeletal injuries among sedentary and physically active adults. Med Sci Sports Exerc. 2002;34(5):838-44.

26. Yeung EW, Yeung SS. A systematic review of interventions to prevent lower limb soft tissue running injuries. Br J Sports Med. 2001;35(6):383-9.

27. Reinking MF. Exercise-related leg pain in female collegiate athletes the influence of intrinsic and extrinsic factors. Am J Sports Med. 2008;34(9):1500-7.

28. Buist I, Bredeweg SW, Lemmink KA, Pepping GJ, Zwerver J, van Mechelen W, et al. Incidence and risk factors of running-related Injuries during preparation for a four-mile recreational running event. $\mathrm{Br} J$ Sports Med. 2010;44(8):598-604 